EPJ Web of Conferences 80, 00002 (2014)

DOI: $10.1051 /$ epjconf/20148000002

(C) Owned by the authors, published by EDP Sciences, 2014

\title{
Low energy constraints and scalar leptoquarks
}

\author{
Svjetlana Fajfer ${ }^{1,2, a}$, Ilja Doršner ${ }^{3, b}$, Nejc Košnik ${ }^{1,2, c}$, and Ivan Nišandžić ${ }^{2, d}$ \\ ${ }^{1}$ Department of Physics, University of Ljubljana, Jadranska 19, 1000 Ljubljana, Slovenia and J. Stefan Insti- \\ tute, Jamova 39, P. O. Box 3000, 1001 Ljubljana, Slovenia \\ ${ }^{2}$ J. Stefan Institute, Jamova 39, P. O. Box 3000, 1001 Ljubljana, Slovenia \\ ${ }^{3}$ Department of Physics, University of Sarajevo, Zmaja od Bosne 33-35, 71000 Sarajevo, Bosnia and Herze- \\ govina
}

\begin{abstract}
The presence of a colored weak doublet scalar state with mass below $1 \mathrm{TeV}$ can provide an explanation of the observed branching ratios in $B \rightarrow D^{(*)} \tau \nu_{\tau}$ decays. Constraints coming from $Z \rightarrow b \bar{b}$, muon $g-2$, lepton flavor violating decays are derived. The colored scalar is accommodated within 45 representation of SU(5) group of unification. We show that presence of color scalar can improve mass relations in the up-type quark sector mass. Impact of the colored scalar embedding in 45-dimensional representation of $\mathrm{SU}(5)$ on low-energy phenomenology is also presented.
\end{abstract}

\section{Introduction}

Recent experimental results coming from LHC and $B$-factories indicate almost an ideal agreement between measured theoretically predicted quantities within the Standard Model. Only few observables disagree on the level of few standard deviations. For example, experimentally observed enhancement of the branching ratios in the $B \rightarrow D\left(D^{*}\right) \tau \bar{v}_{\tau}$ decays with respect to the Standard Model (SM) predictions. Namely, BaBar collaboration has presented the following ratios [2]:

$$
\begin{aligned}
& \mathcal{R}_{\tau / \ell}^{*}=\mathcal{B}\left(B \rightarrow D^{*} \tau v\right) / \mathcal{B}\left(B \rightarrow D^{*} \ell v\right)=0.332 \pm 0.030, \\
& \mathcal{R}_{\tau / \ell}=\mathcal{B}(B \rightarrow D \tau v) / \mathcal{B}(B \rightarrow D \ell v)=0.440 \pm 0.072
\end{aligned}
$$

These results are consistent with previous ones obtained by Belle [3] but higher than the SM values of $\mathcal{R}_{\tau / \ell}^{* \text { SM }}=0.252(3)$ and $\mathcal{R}_{\tau / \ell}^{\mathrm{SM}}=0.296(16)$ with $3.4 \sigma$ significance when the two observables are combined (see Ref. [4]). Among many scenarios of new physics there are viable scenarios where Standard Model is extended by colored scalars. We investigate whether the presence of a light colored scalar state which is a color triplet weak doublet can explain observed discrepancy in $R_{\tau / \ell}^{(*)}$ [1]. Due to its weak doublet nature it modifies relevant couplings in $b \rightarrow c \tau v_{\tau}$ transition and at the same time it affects $Z \rightarrow b \bar{b}$ and lepton electromagnetic moments and $\ell \rightarrow \ell^{\prime} \gamma$ decays. We investigate systematically its impact on all these processes.

\footnotetext{
* talk given by S. Fajfer

a e-mail: svjetlana.fajfer@ijs.si

be-mail: ilja.dorsner@ijs.si

ce-mail: nejc.kosnik@ijs.si

de-mail: ivan.nisandzic@ijs.si
} 


\section{Scalar leptoquark and $B \rightarrow D^{(*)} \tau v_{\tau}$ decays}

The $b \rightarrow c \ell \bar{v}$ transition can be mediated, among other possibilities (see references in [1]), by color triplet scalars (or vectors) with renormalizable leptoquark couplings to the SM fermions. These bosons can have electric charges of $|Q|=1 / 3$ and $|Q|=2 / 3$. The list of all states is given in Table 1 in [1]. Among possible leptoquarks we also discard scalars $(3,1)_{-1 / 3}$, singlet of $S U(2)_{L}$ weak interactions and $(3,3)_{-1 / 3}$, triplet of $S U(2)_{L}$ weak interactions due to their proton destabilization. With this selection we are left with the weak doublet states, which have hypercharge $1 / 6$ or $7 / 6$. There are two states with the electric charge $2 / 3$ and $-1 / 3\left(Q=I_{3}+Y\right)$, with $Y=1 / 6$. One of them might couple to the right handed neutrino and would not interfere with the SM amplitudes. In our approach we do not consider this state, since one has to elaborate on the origin of right handed neutrino. We are left with only one state $\Delta \equiv(3,2)_{7 / 6}$. The leptoquark $\Delta$ interacts with the SM fermions in a following way:

$$
\mathcal{L}=\bar{\ell}_{R} Y \Delta^{\dagger} Q+\bar{u}_{R} Z \tilde{\Delta}^{\dagger} L+\text { H.c. },
$$

where we have used $\tilde{\Delta}=i \tau_{2} \Delta^{*}$ for the conjugated state. This Lagrangian is written in the weak basis and the transition to the mass basis splits Yukawa couplings of the weak doublets to two sets of couplings relevant for the upper and the lower doublet components. The coupling $Y$ represents couplings between charged leptons and down-type quarks while $Z$ connects up-type quarks with charged leptons. We choose basis in which masses of charged leptons and down-type quarks are diagonal and all relative rotations are assigned to neutrinos and up-type quarks and the transition to such basis is achieved by substituting $v_{L} \rightarrow V_{P M N S} v_{L}$ and $u_{L} \rightarrow V_{C K M}^{\dagger} u_{L}$, where $V_{P M N S}$ and $V_{C K M}$ represent Pontecorvo-Maki-Nakagawa-Sakata (PMNS) and Cabibbo-Kobayashi-Maskawa (CKM) mixing matrices, respectively. The two components of colored scalar, i.e., $\Delta^{(2 / 3)}$ and $\Delta^{(5 / 3)}$, then have following interactions with the fermion fields:

$$
\begin{aligned}
& \mathcal{L}^{(2 / 3)}=\left(\bar{\ell}_{R} Y d_{L}\right) \Delta^{(2 / 3) *}+\left(\bar{u}_{R}\left[Z V_{P M N S}\right] v_{L}\right) \Delta^{(2 / 3)}+\text { H.c. }, \\
& \mathcal{L}^{(5 / 3)}=\left(\bar{\ell}_{R}\left[Y V_{C K M}^{\dagger}\right] u_{L}\right) \Delta^{(5 / 3) *}-\left(\bar{u}_{R} Z \ell_{L}\right) \Delta^{(5 / 3)}+\text { H.c. }
\end{aligned}
$$

Existing experimental results teach us that the flavor changing processes within first two generations of quarks and leptons are well fitted with CKM and PMNS parameters. Therefore, we only require nonzero coupling of $\Delta^{(2 / 3)}$ to the third generation $\bar{\tau} b$ but not to $\bar{b} \mu$ or $\bar{b} e$ as indicated by the experimental results suggested by nonobservation of anomalies in $b \rightarrow c \ell \bar{v}$, with $\ell=e, \mu$. We also require that only $c$ quark but not $u$ or $t$ couples to neutrinos. Therefore, we introduce the minimal set of couplings needed to explain the $b \rightarrow c \tau \bar{v}$ branching fraction. These requirements yield

$$
Y=\left(\begin{array}{ccc}
0 & 0 & 0 \\
0 & 0 & 0 \\
0 & 0 & y_{33}
\end{array}\right), \quad Z V_{P M N S}=\left(\begin{array}{ccc}
0 & 0 & 0 \\
z_{21} & z_{22} & z_{23} \\
0 & 0 & 0
\end{array}\right)
$$

The $\Delta^{(5 / 3)}$ Yukawa couplings are related to the above ones through CKM and PMNS rotations that induce CKM-suppressed couplings of $\tau$ to up-type quarks and PMNS-rotated couplings of $c$ quarks to charged leptons. We have

$$
Y V_{C K M}^{\dagger}=y_{33}\left(\begin{array}{ccc}
0 & 0 & 0 \\
0 & 0 & 0 \\
V_{u b}^{*} & V_{c b}^{*} & V_{t b}^{*}
\end{array}\right), \quad Z=\left(\begin{array}{ccc}
0 & 0 & 0 \\
\tilde{z}_{21} & \tilde{z}_{22} & \tilde{z}_{23} \\
0 & 0 & 0
\end{array}\right),
$$

where $\tilde{z}_{2 i}$ are linear combinations of $z_{2 j}$ with $O(1)$ coefficients related to the PMNS matrix elements. One can, at this stage, regard null-entries in $Y$ and $Z$ to be sufficiently small numbers that can thus be neglected in subsequent analyses. 
NP scenarios can be reduced to the effective Lagrangians in which either new vector/axial-vector and tensor currents, or (pseudo)scalar density operators are responsible for the measured discrepancy. There are additional observables that could help single out the class of NP operators preferred by the data [5]. In all these analyses the effective operator contribution was included into decay amplitude on individual basis. The model of LQ mediation we consider here results in scalar/pseudoscalar and tensor contributions simultaneously. Namely, the relevant effective Hamiltonian for semileptonic $b \rightarrow c$ transition induced by the $(3,2)_{7 / 6}$ state is

$$
\mathcal{H}^{(2 / 3)}=\frac{y_{33} z_{2 i}}{2 m_{\Delta}^{2}}\left[\left(\bar{\tau}_{R} v_{i L}\right)\left(\bar{c}_{R} b_{L}\right)+\frac{1}{4}\left(\bar{\tau}_{R} \sigma^{\mu v} v_{i L}\right)\left(\bar{c}_{R} \sigma_{\mu \nu} b_{L}\right)\right],
$$

where $m_{\Delta}$ is the mass of the LQ component with charge $|Q|=2 / 3$ and is also defined as a matching scale for the above Hamiltonian. (In what follows we assume that $\Delta^{(2 / 3)}$ and $\Delta^{(5 / 3)}$ are degenerate in mass.) This means that the appropriate Wilson coefficients of scalar and tensor operators, $g_{S}$ and $g_{T}$, are uniquely determined and correlated. The above leptoquark effective Hamiltonian will affect semileptonic decays with the tau lepton, but contrary to the SM the final state neutrino is not necessarily a $\bar{v}_{\tau}$. The most natural mechanism to enhance $b \rightarrow c \tau \bar{v}$ is to have a constructive interference between the SM and the LQ amplitudes of $b \rightarrow c \tau \bar{v}_{\tau}$, whereas pure leptoquark contributions, producing $\bar{v}_{e}$ and $\bar{v}_{\mu}$ are negligible. This implies that we employ a Hamiltonian that includes the SM as well as the LQ contribution to $b \rightarrow c \tau \bar{v}_{\tau}$ decay

$$
\mathcal{H}=\frac{4 G_{F}}{\sqrt{2}} V_{c b}\left[\left(\bar{\tau}_{L} \gamma^{\mu} v_{L}\right)\left(\bar{c}_{L} \gamma_{\mu} b_{L}\right)+g_{S}\left(\bar{\tau}_{R} v_{L}\right)\left(\bar{c}_{R} b_{L}\right)+g_{T}\left(\bar{\tau}_{R} \sigma^{\mu v} v_{L}\right)\left(\bar{c}_{R} \sigma_{\mu \nu} b_{L}\right)\right]
$$

where the scalar and tensor effective couplings are related to the underlying Yukawa couplings at the matching scale

$$
g_{S}\left(m_{\Delta}\right)=4 g_{T}\left(m_{\Delta}\right) \equiv \frac{1}{4} \frac{y_{33} z_{23}}{2 m_{\Delta}^{2}} \frac{\sqrt{2}}{G_{F} V_{c b}} .
$$

Hadronic (pseudo)scalar and tensor operators in Eq. (7) have anomalous dimensions in QCD and dependence of their matrix elements on the renormalization scale is canceled by the scale dependence of the Wilson coefficients at the leading logarithm approximation. The Wilson coefficients run to the beauty quark scale, i.e., $\mu=m_{b}=4.2 \mathrm{GeV}$, at which the matrix elements of hadronic currents are calculated. As presented in [1] the difference between running of $g_{S}$ and $g_{T}\left(g_{T}\left(m_{L Q}\right)=1 / 4 g_{S}\left(m_{L Q}\right)\right)$ modifies the matching scale relation to $g_{T}\left(m_{b}\right) \simeq 0.14 g_{S}\left(m_{b}\right)$.

For exclusive decay amplitudes for $B \rightarrow D \tau v$ the hadronic matrix element of the vector current is conventionally parametrized by $f_{+}\left(q^{2}\right)$ and $f_{0}\left(q^{2}\right)$ form factors

$$
\left\langle D\left(p_{D}\right)\left|\bar{c} \gamma^{\mu} b\right| \bar{B}\left(p_{B}\right)\right\rangle=\left(p_{B}^{\mu}+p_{D}^{\mu}-\frac{m_{B}^{2}-m_{D}^{2}}{q^{2}} q^{\mu}\right) f_{+}\left(q^{2}\right)+\frac{m_{B}^{2}-m_{D}^{2}}{q^{2}} q^{\mu} f_{0}\left(q^{2}\right),
$$

where $p_{B, D}^{\mu}$ are four vectors of momenta of $B$ and $D$ mesons and $q^{\mu}=p_{B}^{\mu}-p_{D}^{\mu}$. The presence of the tensor operator in Eq. (8) requires inclusion of an additional form factor $f_{T}\left(q^{2}\right)$, defined as

$$
\left\langle D\left(p_{D}\right)\left|\bar{c} \sigma^{\mu v} b\right| \bar{B}\left(p_{B}\right)\right\rangle=-i\left(p_{B}^{\mu} p_{D}^{v}-p_{D}^{\mu} p_{B}^{v}\right) \frac{2 f_{T}\left(q^{2}\right)}{m_{B}+m_{D}} .
$$

The matrix element of a scalar operator is related to $f_{0}\left(q^{2}\right)$ form factor $\langle D|\bar{c} b| \bar{B}\rangle=\frac{m_{B}^{2}-m_{D}^{2}}{m_{b}-m_{c}} f_{0}\left(q^{2}\right)$, where $m_{b}$ and $m_{c}$ are masses of $b$ and $c$ quarks in $\overline{M S}$ scheme at the scale $\mu=m_{b}$ [6]. Using these connections 
the differential branching ratio can be expressed by form factors $f_{+}\left(q^{2}\right), f_{0}\left(q^{2}\right)$ and $f_{T}\left(q^{2}\right)$ as shown in Eq. (3.8) of [1]. In our numerical calculations the ratio $f_{T}\left(q^{2}\right) / f_{+}\left(q^{2}\right)=1.03(1)$ is evaluated in the model of Ref. [7]. In the heavy quark limit this ratio is $f_{T}\left(q^{2}\right) / f_{+}\left(q^{2}\right)=1$, as the form factors are equally related to the Isgur-Wise function, $f_{+}\left(q^{2}\right)=f_{T}\left(q^{2}\right)=\frac{m_{B}+m_{D}}{2 \sqrt{m_{B} m_{D}}} \xi(w)$. We employ following parametrization of vector form factors [8-10]

$$
f_{+}\left(q^{2}\right)=\left.\frac{G_{1}(w)}{R_{D}}\right|_{w\left(q^{2}\right)}, \quad f_{0}\left(q^{2}\right)=\left.R_{D} \frac{1+w}{2} G_{1}(w) \frac{1+r_{D}}{1-r_{D}} \Delta(w)\right|_{w\left(q^{2}\right)},
$$

where the constant $R_{D}$ and the new kinematic variable $w$ are given as

$$
R_{D}=\frac{2 \sqrt{m_{B} m_{D}}}{m_{B}+m_{D}}, \quad w=\frac{m_{B}^{2}+m_{D}^{2}-q^{2}}{2 m_{B} m_{D}},
$$

and $r_{D}$ denotes the ratio of masses of $D$ and $B$ mesons. Lattice estimate of the function $\Delta(w)$ is consistent with constant value $\Delta(w)=0.46 \pm 0.02$ as described in [1].

The decay $B \rightarrow D^{*} \tau \bar{\nu}_{\tau}$ offers additional tests of the SM and NP due to the vector meson state $D^{*}$ [5]. In the case of light lepton in the final state, one vector and two axial form factors are present in the decay amplitude. If $\tau$ is in the final state an additional form factor $A_{0}\left(q^{2}\right)$ appears. The mediation of the $(3,2)_{7 / 6}$ leptoquark induces effective Lagrangian containing the tensor operator that requires knowledge of tensor form factors. Following notation of Ref. [5], the polarization four-vectors of the final state leptons and $D^{*}$ vector meson are denoted by $\tilde{\epsilon}_{\mu}(\lambda)$ and $\epsilon_{\mu}\left(\lambda_{D^{*}}\right)$, respectively. Polarizations take the following values: $\lambda=0, \pm, t$ and $\lambda_{D^{*}}= \pm, 0$. Here $t$ stands for the time-like polarization vector. Standard parametrization of vector and axial hadronic matrix elements for the $B \rightarrow D^{*}$ transition is given by

$$
\begin{aligned}
\left\langle D^{*}\left(p_{D^{*}}, \epsilon\right)\left|\bar{c} \gamma_{\mu} b\right| B\left(p_{B}\right)\right\rangle= & \frac{2 i V\left(q^{2}\right)}{m_{B}+m_{D^{*}}} \epsilon_{\mu \nu \alpha \beta} \epsilon^{* v} p_{B}^{\alpha} p_{D^{*}}^{\beta}, \\
\left\langle D^{*}\left(p_{D^{*}}, \epsilon\right)\left|\bar{c} \gamma_{\mu} \gamma_{5} b\right| B\left(p_{B}\right)\right\rangle= & 2 m_{D^{*}} A_{0}\left(q^{2}\right) \frac{\epsilon^{*} \cdot q}{q^{2}} q_{\mu}+\left(m_{B}+m_{D^{*}}\right) A_{1}\left(q^{2}\right)\left(\epsilon_{\mu}^{*}-\frac{\epsilon^{*} \cdot q}{q^{2}} q_{\mu}\right) \\
& -A_{2}\left(q^{2}\right) \frac{\epsilon^{*} \cdot q}{m_{B}+m_{D^{*}}}\left(\left(p_{B}+p_{D^{*}}\right)_{\mu}-\frac{m_{B}^{2}-m_{D^{*}}^{2}}{q^{2}} q_{\mu}\right) .
\end{aligned}
$$

For the parametrization of the tensor hadronic matrix elements, we follow Ref. [11] and relations in heavy quark limit among tensor, vector and axial form factors as presented in [1].

The branching ratio is calculated after integration over $q^{2}$ and angle $\theta_{l}$. We constrain the allowed values of tensor and scalar Wilson coefficients using BaBar's measurements of the ratios $\mathcal{R}_{\tau / \ell}=$ $\mathcal{B}(\mathcal{B} \rightarrow \mathcal{D} \tau v) / \mathcal{B}(\mathcal{B} \rightarrow \mathcal{D} \ell v)$ and $\mathcal{R}_{\tau / \ell}^{*}=\mathcal{B}\left(\mathcal{B} \rightarrow \mathcal{D}^{*} \tau v\right) / \mathcal{B}\left(\mathcal{B} \rightarrow \mathcal{D}^{*} \ell v\right)$ as shown in Fig. 1, where the result of fit to both ratios is shown. We derive $1 \sigma$ range for the Wilson coefficient $g_{S}$ at the low scale

$$
g_{S}\left(m_{b}\right)=-0.37_{-0.07}^{+0.10},
$$

where we have assumed $g_{S}$ to be real in estimating the error bars. The coupling $g_{S}$ at the matching scale is rescaled by factor 0.64 with respect to the above value due to QCD corrections as explained in the text. We have compared the $q^{2}$ distributions of decay widths for $B \rightarrow D \tau \bar{v}$ and $B \rightarrow D^{*} \tau \bar{v}$ for our best fit point, $g_{S}=-0.37$, against the experimental bin values presented in [2]. The effect of leptoquark does not generate observable $q^{2}$-dependent features. The existing level of experimental and hadronic uncertainties imply that there is no possibility to discern a signal of leptoquark from the SM contribution, based on observation of a single bin. The discrepancy becomes more pronounced after integrated over large range of $q^{2}$. 


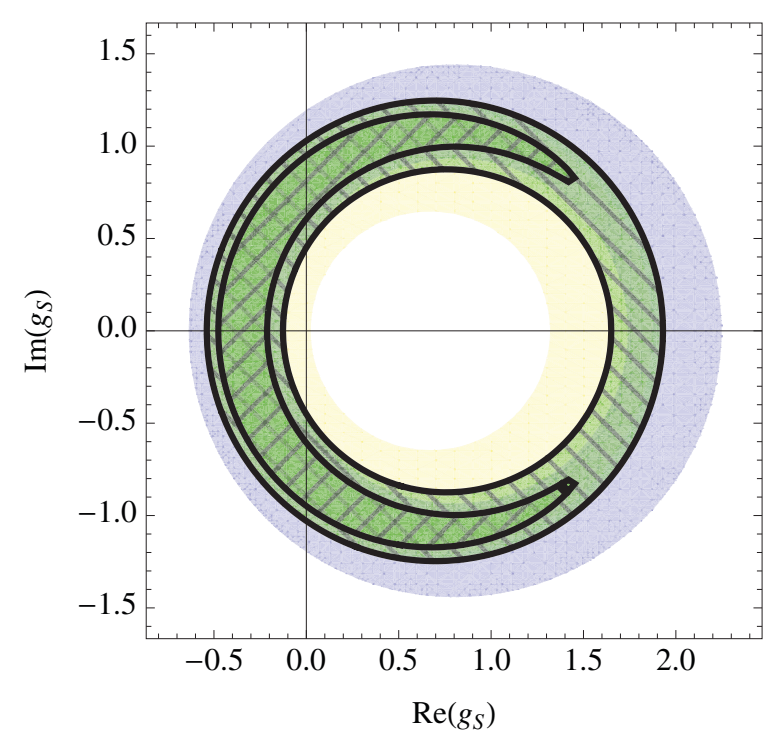

Figure 1. Values of the scalar Wilson coefficient $g_{S}\left(m_{b}\right)\left(g_{T}\left(m_{b}\right) \simeq 0.14 g_{S}\left(m_{b}\right)\right)$ consistent at $2 \sigma$ with BaBar Collaboration's measurements of ratios $\mathcal{R}(D)$ (bright ring) and $\mathcal{R}\left(D^{*}\right)$ (darker ring). The $1 \sigma(2 \sigma)$ region, fitted to the two constraints, is doubly (singly) hatched.

\section{Constraints from $Z \rightarrow b \bar{b}$ and lepton electromagnetic moments}

The same couplings appear in other observables. There is well known SM prediction - experimental result discrepancy in $Z \rightarrow b \bar{b}$ (see e.g.[12]). Standard parameterization of the $Z b \bar{b}$ renormalizable coupling is adopted $\mathcal{L}_{Z b \bar{b}}=\frac{g}{c_{W}} Z^{\mu} \bar{b} \gamma_{\mu}\left[\left(g_{L}^{b}+\delta g_{L}^{b}\right) P_{L}+\left(g_{R}^{b}+\delta g_{R}^{b}\right) P_{R}\right] b, S U(2)$ coupling is denoted by $g, c_{W}$ is the cosine of the Weinberg angle and $P_{L, R}=\left(1 \pm \gamma_{5}\right) / 2$ are the chiral projectors. At the SM tree-level, the couplings are $g_{L}^{b 0}=-1 / 2+s_{W}^{2} / 3$ and $g_{R}^{b 0}=s_{W}^{2} / 3$. Higher-order electroweak corrections that are contained within $g_{L, R}^{b}$ get largest contributions from top quark in loops. A recent electroweak fit that includes updated theoretical predictions and new results from LHC points to tensions in the $Z \rightarrow b \bar{b}$ observables reaching above $2 \sigma$ significance in $R_{b}$ and $A_{F B}^{b}$ [12]. The shifts with respect to the $\mathrm{SM}$ values of couplings are then $\delta g_{L}^{b}=0.001 \pm 0.001$ and $\delta g_{R}^{b}=(0.016 \pm 0.005) \cup(-0.17 \pm 0.005) . \Delta^{(2 / 3)}$ component possesses a possibly large coupling $y_{33}$ to $b \tau$ pair that contributes to $Z \rightarrow b \bar{b}$ amplitude at order $\left|y_{33}\right|^{2}$ and thus allows one to constrain it directly. The LQ correction to the left-handed coupling is

$$
\delta g_{L}^{b}\left(y_{33}\right)=\frac{\left|y_{33}\right|^{2}}{384 \pi^{2}}\left[g_{0}(x)+s_{W}^{2} g_{2}(x)\right]
$$

where $x=m_{\Delta}^{2} / m_{Z}^{2}$, and we take $m_{Z}=91.2 \mathrm{GeV}$ and $s_{W}^{2}=0.231$. The shift $\delta g_{L}^{b}$ might receive the imaginary part due to on-shell $\tau$ leptons. The constraint from the electroweak fit is sensitive to the interference term between the approximately real $g_{L}^{b}$ and complex $\delta g_{L}^{b}\left(y_{33}\right)$, and therefore only the real part of $\delta g_{L}^{b}\left(y_{33}\right)$ enters the prediction. The constraint $\delta g_{L}^{b}\left(y_{33}\right)=0.001 \pm 0.001$ leads to

$$
\left|y_{33}\right|_{\text {central }}=1.57+2.86 \frac{m_{\Delta}}{500 \mathrm{GeV}}
$$

as explained in [1]. For $m_{\Delta}$ above $300 \mathrm{GeV}$ large portion of preferred $\left|y_{33}\right|$ range lies within the nonperturbative regime. In order to maintain a predictable setup we assume that coupling $y_{33}$ is perturbative, i.e., $\left|y_{33}\right|<\sqrt{4 \pi}$.

Additional constraints are available from a number of lepton electromagnetic moments appearing in $\ell \ell \gamma$ vertex. The lepton vertex with electromagnetic field will be modified by penguin diagrams 
involving virtual exchanges of $\Delta^{(5 / 3)}$ and charm quark. These contributions enter into muon $g-2$. The muon anomalous moment gets shifted due to new contribution of $\Delta^{(5 / 3)}$ in the loop. We determined the allowed $1 \sigma$ range from the condition $\chi^{2}-\chi_{S M}^{2} \leq 1$ that translates to a constraint $\left|\delta a_{\mu}\right|<10.9 \times 10^{-11}$ or put in terms of $\tilde{z}_{22}$ (see Fig. 3 in [1]) $\left|\tilde{z}_{22}\right|<0.51 \frac{m_{\Delta}}{500 \mathrm{GeV}}$.

The $\tau$ electric dipole moment arises at a loop level with the helicity flip on the virtual charm quark and consequently both penguin diagrams are finite (for details see [1]). At the moment, the best bounds from Belle experiment are orders of magnitude too weak to directly probe the parameter range, preferred by $B \rightarrow D^{(*)} \tau \bar{v}$. We then found from bound $\left|\tilde{z}_{23} y_{33}\right|<4 \pi$ that the upper bound on tau EDM in the perturbative setting is $\left|d_{\tau}\right|<2.6 \times 10^{-21} \mathrm{ecm}$.

The additional constraints might be derived from the lepton flavor violating $\ell \rightarrow \ell^{\prime} \gamma$ decays The decay $\tau \rightarrow \ell \gamma$ is mediated by a loop diagram with $\Delta^{(5 / 3)}$ scalar and a charm quark. The best experimental bounds on LFV radiative decays of $\tau$ were presented by BaBar collaboration in Ref. [13]. Experimental bounds on branching ratios $\mathrm{B}(\tau \rightarrow \mathrm{e} \gamma)<3.3 \times 10^{-8}$ and $\mathrm{B}(\tau \rightarrow \mu \gamma)<4.4 \times 10^{-8}$ (both at $90 \%$ C.L.) severely constrain the combination of couplings coming from LQ contributions. The experimental upper limits for $\mu \rightarrow e \gamma$ branching ratio are orders of magnitude more stringent and in conjuction with the small width of the muon they compensate for the $m_{\mu}$ suppression in sensitivity. We rely on the latest result from the MEG experiment obtained from data collected in years 2009$2011[14], \mathrm{B}(\mu \rightarrow \mathrm{e} \gamma)<5.7 \times 10^{-13}$, at $90 \%$ C.L. .

\section{$4(3,2)_{7 / 6}$ in GUT framework}

The leptoquark $\Delta \equiv(3,2)_{7 / 6}$ can be naturally accommodated within a framework of matter unification (see references $[53,54]$ in [1]). In our study we wanted to investigate whether a particular low-energy ansatz with Yukawa couplings is compatible with the idea of grand unification. Within this approach $\Delta$ is one of states within the 45-dimensional representation. Some of the $\Delta$ couplings to matter are proportional to $Z$. The $S U(5)$ contractions relevant for these Yukawa couplings are $\left(Y_{1}\right)_{i j} \mathbf{1 0}_{i} \overline{\mathbf{5}}_{j} \mathbf{4 5}$ and $\left(Y_{3}\right)_{i j} \mathbf{1 0}_{i} \overline{\mathbf{5}}_{j} \mathbf{5}$ where $\mathbf{1 0}_{i}$ and $\overline{\mathbf{5}}_{i}$ together comprise an entire generation of fermions. $Y_{1}$ and $Y_{3}$ are, at this stage, arbitrary $3 \times 3$ matrices in flavor space with $i, j(=1,2,3)$ being corresponding generation indices.

We denoted unitary transformations of the down-type quark fields to be $D_{L}$ and $D_{R}$, where subscripts $L$ and $R$ are related to appropriate chirality. These rotations take the down-type quark fields from a flavor into a mass eigenstate basis. For the up-type quark (charged lepton) sector we similarly used $U_{L}$ and $U_{R}\left(E_{L}\right.$ and $\left.E_{R}\right)$ to be appropriate unitary matrices. Our assumption for neutrinos was that they are Majorana particles and accordingly denote unitary matrix that defines the neutrino mass eigenstates with $N$.

Following details given in [1], after we imposed the ansatz of Eq. (5) on possible Yukawa couplings $\left(Y_{1}\right)_{i j} \mathbf{1 0}_{i} \overline{\mathbf{5}}_{j} \mathbf{4 5}$ and $\left(Y_{3}\right)_{i j} \mathbf{1 0} \overline{\mathbf{5}}_{j} \mathbf{5}$ at the GUT scale, we found two relations that connect fermion mass matrices of down-type quarks and charged leptons with the original Yukawa couplings:

$$
2 M_{D}^{\mathrm{diag}} D_{R}^{T}=-2 Y_{1} v_{45}-Y_{3} v_{5}, 2 E_{R} M_{E}^{\mathrm{diag}}=6 Y_{1} v_{45}-Y_{3} v_{5} .
$$

Here, $M_{D}^{\text {diag }}\left(M_{E}^{\text {diag }}\right)$ is a diagonal mass matrix for down-type quarks (charged leptons) and we take both vacuum expection value of representations 5 and $45\left(v_{5}\right.$ and $\left.v_{45}\right)$ to be real. Note that the relations in question contain only the right-handed unitary transformations $D_{R}$ and $E_{R}$. We proceed by identifying a connection between $Y_{1}$ and $Z$ to be $Y_{1}=-U_{R} Z$. This, then, leads us to the following matrix equation $M_{D}^{\text {diag }} D_{R}^{T}-E_{R} M_{E}^{\text {diag }}=4 U_{R} Z v_{45}$. We have checked numerically if our ansatz is compatible with the idea of $S U(5)$ grand unification (the details of this work are presented in [1]). We found that numerically 


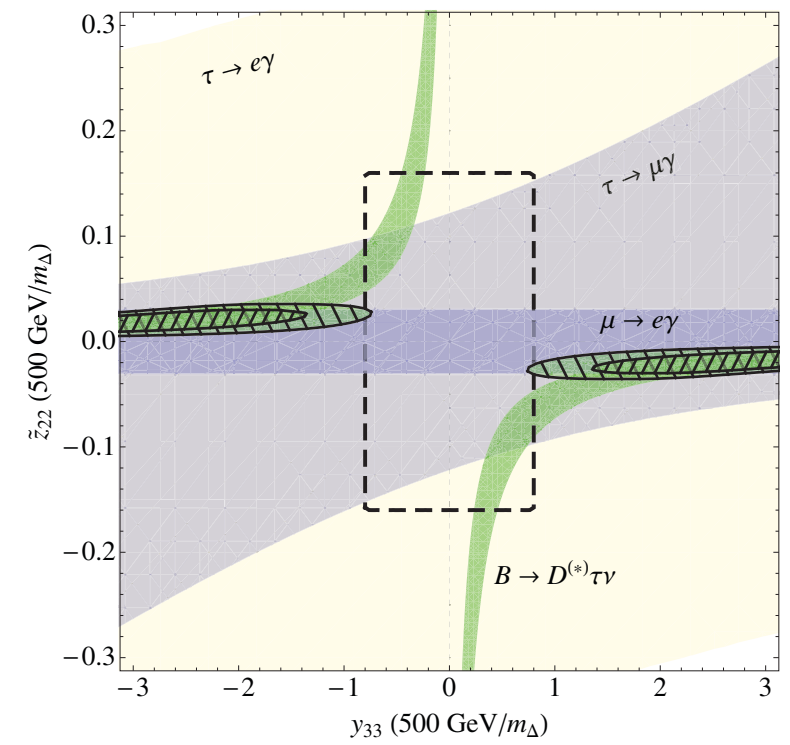

Figure 2. Constraints on the couplings to $b \tau$ $\left(y_{33}\right)$ and to $c \mu\left(\tilde{z}_{22}\right)$ coming from the $1 \sigma$ region of $\mathcal{R}_{\tau / \ell}^{(*)}$ (thin hyperbolic region), $90 \%$ CL upper bounds on $\mu \rightarrow e \gamma, \tau \rightarrow \mu \gamma$ and $\tau \rightarrow e \gamma$. Dashed frame represents the region where couplings remain perturbative all the way to the GUT scale, as explained in the text. Doubly (singly) hatched area is allowed at $1 \sigma(2 \sigma)$.

there exists a hierarchical relation among $\tilde{z}_{21}, \tilde{z}_{22}$ and $\tilde{z}_{23}$ that mimics mass hierarchy in the down-type quark and the charged lepton sectors with $\tilde{z}_{23}$ being a dominant element:

$$
\tilde{z}_{21}: \tilde{z}_{22}: \tilde{z}_{23}=0.024: 0.32: 1 \text {. }
$$

We point out that these results are completely independent from the up-type quark and the neutrino sectors, where CKM and PMNS mixing parameters reside, respectively.

The hierarchy in the $Z$ matrix, originating from quark-mass hierarchy, enables us to reduce the parameter set of the model to two independent Yukawa couplings. One of these has to be $y_{33}$ while we choose $\tilde{z}_{22}$, the coupling of $\Delta^{(5 / 3)}$ scalar to $c \mu$ pair, to be the remaining free parameter. The rest of the couplings are determined, either from the hierarchical pattern (19) or using properties of PMNS matrix (5). With the help of the PMNS rotation connecting $\tilde{z}_{2 j}$ and $z_{2 k}$ couplings we deduced $z_{23}=\tilde{z}_{2 k} V_{k 3} \approx \tilde{z}_{22} c_{13}\left(s_{23}+3.22 c_{23}\right)$. The numerical factor 3.22 comes from the hierarchy between $\tilde{z}_{2 k}$ couplings. $V_{i j}, s_{i j}$ and $c_{i j}$ denote the PMNS matrix elements and the mixing angles that parameterize it, respectively. Using the $3 \sigma$ ranges for mixing angles from a recent PMNS fit as described in [1] $z_{23}=\omega \tilde{z}_{22}, \quad 2.63<\omega<3.17$. Effect of the aforementioned experimental constraints on $(3,2)_{7 / 6}$ with the minimal Yukawa texture (5), additionally restricted by the pattern of fermion masses, is shown on Fig. 2. As expected, the central role is played by the constraint on $g_{S}$, although $\tau \rightarrow \mu \gamma$ reduces the parameter space remarkably. Due to suppressed coupling to $e$, sensitivity of $\tau \rightarrow e \gamma$ and $\mu \rightarrow e \gamma$ observables is reduced, however, the latter overcomes this suppression by a very stringent experimental upper bound and therefore has the most important role next to constraint on $g_{S}$. An order of magnitude improvement on the experimental bound on $\mu \rightarrow e \gamma$ would cause tension with the $R\left(D^{(*)}\right)$ observables, and smaller values of the $g_{S}$ coupling would be preferred. Note that only the $2 \sigma$ region (hatched in Fig. 2) is overlapping with the region where $y_{33}$ is perturbative all the way to the GUT scale. We mention in passing that for mass $200 \mathrm{GeV}<m_{\Delta}<1 \mathrm{TeV}$ all predictions are approximately invariant under rescaling of couplings and $m_{\Delta}$ by same factor, as indicated on the axes of Fig. 2.

The $2 \sigma$ region at $m_{\Delta}=500 \mathrm{GeV}$ in the $y_{33}-\tilde{z}_{22}$ plane implies the following bounds $\left|y_{33}\right|>0.74$, $\left|\tilde{z}_{22}\right|<0.037$. Region that satisfies perturbativity of the couplings all the way to the GUT scale, 
outlined by dashed frame in Fig. 2, restricts the above $2 \sigma$ ranges to

$$
0.74<\left|y_{33}\right|<0.80, \quad 0.021<\left|\tilde{z}_{22}\right|<0.032 \text {. }
$$

Furthermore, these bounds might generate limits on allowed values of $v_{45}$. Relaying on the GUT model we derive bounds on the $v_{45}: f_{\mathrm{RGE}} 5.0 \mathrm{GeV}<v_{45}<f_{\mathrm{RGE}} 7.6 \mathrm{GeV}$.

Our approach combining constraints from the low energy phenomenology and the constraints coming from the GUT setup results in a number of predictions. An obvious independent test of the $b \rightarrow c \tau \bar{v}$ process would be the decay $B_{c} \rightarrow \tau \bar{v}_{\tau}$ that probes the same couplings as the semileptonic decay, which branching ratio of $B_{c} \rightarrow \tau \bar{v}_{\tau}$ might be enhanced for 1 to 2 orders of magnitude. Due to the special interaction of the $(3,2)_{7 / 6}$ leptoquark to $\tau b$ pair and allowing it to couple to charm quark and all three neutrinos we can successfully explain the observed deviations in semileptonic $b \rightarrow c$ transitions. This leptoquark state causes lepton flavor violating decays of the tau leptons and muons and rare decays of top quark to charm quark and a pair of tau leptons.

Acknowledgments I. D. acknowledges the SNSF support through the SCOPES project No. IZ74Z0_137346. This work is supported in part by the Slovenian Research Agency.

\section{References}

[1] I. Doršner, S. Fajfer, N. Košnik and I. Nišandžić, JHEP 1311 (2013) 084

[2] J. P. Lees et al. [The BaBar Collaboration], Phys. Rev. D 88 (2013) 7, 072012

[3] A. Matyja et al. [Belle Collaboration], Phys. Rev. Lett. 99 (2007) 191807

[4] S. Fajfer, J. F. Kamenik, I. Nisandzic and J. Zupan, Phys. Rev. Lett. 109 (2012) 161801

[5] S. Fajfer, J. F. Kamenik and I. Nisandzic, Phys. Rev. D 85 (2012) 094025 [arXiv:1203.2654 [hep$\mathrm{ph}]]$.

[6] D. Becirevic, N. Kosnik and A. Tayduganov, Phys. Lett. B 716, 208 (2012) [arXiv:1206.4977 [hep-ph]].

[7] D. Melikhov and B. Stech, Phys. Rev. D 62 (2000) 014006

[8] A. F. Falk and M. Neubert, Phys. Rev. D 47 (1993) 2965

[9] A. F. Falk and M. Neubert, Phys. Rev. D 47 (1993) 2982

[10] M. Neubert, Phys. Rev. D 46 (1992) 2212.

[11] P. Biancofiore, P. Colangelo and F. De Fazio, Phys. Rev. D 87 (2013) 7, 074010

[12] B. Batell, S. Gori and L. T. Wang, JHEP 1301 (2013) 139

[13] B. Aubert et al. [BaBar Collaboration], Phys. Rev. Lett. 104 (2010) 021802

[14] J. Adam et al. [MEG Collaboration], Phys. Rev. Lett. 110 (2013) 20, 201801 